\title{
THE ROLE OF LIGHT SCATTER IN THE DEGRADATION OF VISUAL PERFORMANCE BEFORE AND AFTER Nd:YAG CAPSULOTOMY
}

\author{
R. R. GOBLE ${ }^{1}$, D. P. S. O'BRART ${ }^{1}$, C. P. LOHMANN ${ }^{1}$, F. FITZKE ${ }^{2}$ and J. MARSHALL ${ }^{1}$ \\ London
}

\begin{abstract}
SUMMARY
The aim of this study was to determine whether capsulotomy size influences visual performance. Snellen visual acuity and forward light scatter (light scattered towards the patient's retina, but out of the focussed retinal image) measurements using simple computer graphics based on van den Berg's technique were used to measure visual performance. Twelve patients were studied: 4 had small central capsulotomies through undilated pupils and 8 had wide capsulotomies through dilated pupils. The two groups were matched for age and pre-laser Snellen acuity. Following treatment, both groups had equal improvements in Snellen acuity. There was only a significant $(\boldsymbol{p}<\mathbf{0 . 0 0 1 )}$ improvement in forward light scatter readings in the group who received wide capsulotomies. Measurements of forward light scatter are more sensitive than Snellen acuity testing in demonstrating loss of visual performance in patients with media opacities. It is recommended that pupils are dilated prior to Nd:YAG capsulotomy if forward light scatter from capsule remnants and the consequent glare disability are to be minimised.
\end{abstract}

In the last decade, extracapsular cataract extraction with posterior chamber intraocular lens insertion has become the treatment of choice for patients with cataracts. Only $2-4 \%$ of routine procedures are now performed by the intracapsular technique. ${ }^{1.2}$ It is estimated that in excess of 1 million implants are inserted annually in the United States of America and approximately 100000 in the United Kingdom. ${ }^{1.3}$ Posterior capsule opacification is a common sequel to otherwise successful surgery and $\mathrm{Nd}$ :YAG capsulotomy is the accepted method of treatment as it has fewer attendant complications than surgical capsulotomy. ${ }^{4}$ Patient satisfaction with this technique is

From: 'Department of Ophthalmology, St Thomas' Hospital, London SE1 7EH, UK. ${ }^{2}$ Department of Visual Science, Institute of Ophthalmology, London EC1V 9AT, UK.

Correspondence to: Mr R. R. Goble, Registrar in Ophthalmology, Sussex Eye Hospital, Brighton, Sussex BN2 5BF, UK. high but some complain of glare around point sources of light at night, and a few consider it sufficiently disturbing to cause them to refrain from driving at night. No systematic study has been undertaken to evaluate the effects that surgical parameters such as capsulotomy size and implant chipping may have on the patient's post-operative visual performance.

In many clinical practices, the only assessment of visual performance is Snellen acuity testing. Few centres perform variable contrast acuity measurements although several such tests are now readily available. ${ }^{5}$ In practice if a patient complains of visual deterioration or the corrected Snellen acuity is noted to have worsened, then ocular examination with slit lamp biomicroscopy will ensue. The slit lamp image of capsular or any other media opacities relies upon light either being reflected or scattered backwards (towards the observer). The cells which make up capsular opacities are of a size and morphology to cause light scatter in all directions.

Recent studies on transient loss of corneal transparency subsequent to excimer laser surgery have demonstrated that the slit lamp image gives no indication as to the degree of the disturbance in the patient's vision. ${ }^{6}$ Such studies have further demonstrated that there was a very good correlation between loss of low contrast visual acuity and the magnitude of light scattered forwards into the patient's eye. This light is scattered towards the patient's retina, but out of the focussed retinal image. It is assumed that the cellular proliferation over the posterior capsule is responsible for the production of light scatter and therefore maximal clearance of such cells from the visual axis should give the optimal visual result.

To investigate this concept we have assessed two groups, one of which received small central capsulotomies and the other wide capsulotomies. We have used the software developed during the excimer studies in order to measure the magnitude of forward-scattered light in

Eye (1994) 8, 530-534 C 1994 Royal College of Ophthalmologists 
pseudophakic patients. Individuals were assessed both before and after $\mathrm{Nd}$ :YAG laser capsulotomy.

\section{MATERIALS AND METHODS}

\section{Patients}

Patients were selected from those referred for Nd:YAG capsulotomy. In order to minimise fluctuations in results resulting from collateral ocular pathology, the following exclusion criteria were employed: media opacities other than opacified posterior capsules (e.g. anterior capsule remnants), pre-existing macular disease (e.g. age-related macular degeneration) or optic nerve disease (e.g. glaucoma), amblyopia or decentred intraocular lenses (e.g. dialling holes or optic edges within or adjacent to the pupil). This helped to eliminate other sources of forward light scatter/reduced visual performance. The actual test to measure forward light scatter was designed for a population of young myopes and a proportion of the elderly population in this study were unfortunately unable to perform or apparently to understand this test and had to be excluded.

Twelve eyes ( 11 patients) aged between 60 and 82 years were assigned to one of two groups. Group A consisted of 4 eyes of 4 patients who underwent small central capsulotomies which were fashioned through undilated pupils (average age 74 years). Group B comprised 8 eyes of 7 patients in whom wide capsulotomies were performed after pupil dilatation (average age 71 years). All had been operated on at St Thomas' Hospital 6-34 months previously. The implants used in the two groups were all manufactured by Kabi Pharmacia and the details are given in Table I.

\section{Capsulotomy Technique}

All capsulotomies were undertaken by R.R.G. or D.P.S.O'B. using a Lasag Microruptor MR-2G, operating in the Fundamental Mode with a 12 nanosecond pulse and focussed with the aid of a corneal contact lens. The technique for laser capsulotomy was standardised for each group and is given below.

In group A the pupil was not dilated and the exposures were delivered initially over a vertical line typically extending 2-3 $\mathrm{mm}$ in length and commencing inferiorly. If capsule flaps remained within the pupil, the opening in the capsule was extended with repeated exposures in the horizontal meridian. The average number of pulses was 41 , average pulse energy was $1.2 \mathrm{~mJ}$ and the average total energy required was $49 \mathrm{~mJ}$.

In group B, the patient's pupil was dilated with guttae tropicamide $1 \%$ and, although the same exposure

Table I. Kabi Pharmacia intraocular lenses (IOL) used in the study population

\begin{tabular}{lllc}
\hline IOL & Group A & Group B & IOL characteristics \\
\hline $720 \mathrm{~A}$ & 3 patients & 4 patients & $6.5 \mathrm{~mm}$ optic, all PMMA \\
$725 \mathrm{~A} / \mathrm{C}$ & 1 patient & 3 patients & $7.0 \mathrm{~mm}$ optic, all PMMA \\
U137L & None & 1 patient & $7.0 \mathrm{~mm}$ optic, 3-piece \\
\hline
\end{tabular}

geometry was employed, the vertical exposure was increased to 5-6 $\mathrm{mm}$ and the horizontal exposures to the capsulotomy edge often needed to be multiple. If capsule flaps remained adherent to the implant, a small circumferential exposure at the capsulotomy edge usually sufficed to remove them from the central area. The average number of pulses was 88 , the average pulse energy was $1.0 \mathrm{~mJ}$ and the average total energy was $94 \mathrm{~mJ}$.

\section{Measurement of Snellen Acuity}

Following retinoscopy and appropriate refractive correction with trial lenses, the patients viewed an internally illuminated Snellen acuity wall chart at $6 \mathrm{~m}$. The eye not being tested was occluded. All patients were tested under standard clinic conditions.

\section{Measurement of Forward Light Scatter}

The method employed in this study was a modification of van den Berg's technique, using a computer graphics video display and computerised data collection and analysis. $^{7.8}$ The retinal light distribution from any visual environment consists of two components: the focussed image of the object of interest and unfocussed light from the environment, the latter producing a more homogeneous background level upon which the focussed image is superimposed. If the background level increases relative to the image of interest, then contrast is lost and acuity deteriorates. Any component which scatters light out of the focal point into the background serves to decrease the contrast between the two components and degrade vision. Van den Berg's test utilises this principle by surrounding a central target with an annulus of background illumination and by comparing the contrast between the two a measure of intraocular scatter can be obtained.

Details of the computer technique have been described in full elsewhere. ${ }^{7}$ Stimuli were generated by a Viglen IV/33 computer on a Viglen CA 1428B high-quality video graphics display. The computer screen was viewed monocularly at a fixed distance of $28 \mathrm{~cm}$ from eye to monitor. The central fixation stimulus consisted of a circle with a radius of $1^{\circ}$. It was set at a level termed the match luminance. This luminance was a random value brighter than that of the background. The central stimulus flickered at $7.5 \mathrm{~Hz}$ between the match level and the background level. The match level was under the control of the patient and could be increased or decreased by depressing two buttons on the computer keyboard. The patient's task was to adjust the contrast between the match luminance and the background in order to minimise or abolish the flicker. Once this had been achieved a button was depressed and the datum recorded. The computer recorded the match luminance value and the difference between the match and background luminance values which were converted to contrast using the Michelson contrast definition. ${ }^{9}$ The match level was then automatically reset to a new random level and the process was repeated ten times.

The test was divided into two parts: the first without straylight and the second with a straylight annulus which 
flickered in counterphase to the match stimulus. The straylight contributed to the luminance of the central test target due to forward light scatter and this increased the patient's error in attempting to abolish the flicker. The difference between the two parts of the test provided a measure of the level of forward-scattered light experienced by the patient.

\section{Clinical Assessment after Capsulotomy}

Measurements of best corrected Snellen acuity and forward light scatter were undertaken immediately prior to and a fortnight after capsulotomy. On review after laser treatment, patients were examined on the slit lamp without mydriasis and the presence of anterior chamber flare or cells, capsule remnants within the pupil and evidence of intraocular lens 'pitting' by the laser exposures was noted.

\section{RESULTS}

\section{Clinical Assessment}

No eye had clinically apparent uveitis on review after laser treatment. Residual capsule flaps were noted in all 4 eyes from group A, some of which were fairly large. Two eyes from group B had small capsule tags remaining in the pupillary zone. Two eyes from group A $(n=4)$ had central pitting and 2 from group $\mathrm{B}(n=8)$ had minimal peripheral pitting of the implant by the laser.

\section{Snellen Acuity}

The results of Snellen acuity measurement are shown in Fig. 1. Both groups had equal improvements in acuity following capsulotomy with $75 \%$ of patients displaying improvements of at least one line. Three patients had no change in their acuities post-laser ( 1 from group A and 2 from group B). There was no significant difference between the two treatment groups, as can be seen by the following average acuity values:

\begin{tabular}{|c|c|}
\hline Group A pre-laser & Group B pre-laser \\
\hline average: & average: \\
\hline roup A post-laser & Group B post-laser \\
\hline average: & average: \\
\hline
\end{tabular}

\section{Forward Light Scatter}

Clear differences were observed between the two groups in measurements of forward light scatter. Group A showed little improvement (Fig. 2). Group B displayed a marked improvement which was statistically (unpaired Students $t$-test) significant at $p<0.001$ (Fig. 3). The average percentage error scores were:

\begin{abstract}
Group A pre-laser. without straylight: with straylight:
\end{abstract} $45.8 \%$ without straylight: $59.5 \%$ with straylight: $38.3 \%$ $55.1 \%$

Group A post-laser without straylight: with straylight:

Group B post-laser

$40.4 \%$ without straylight: $14.0 \%$ $57.7 \%$ with straylight: $\quad 19.3 \%$

\section{DISCUSSION}

The incidence of pseudophakic patients developing pos- terior capsular opacification varies in the literature between $9 \%$ and $51 \%$, depending on the criteria used for diagnosis, the length of follow-up and the surgical technique. ${ }^{10.11}$ Some attempts have been made to relate patients' visual disability to the morphological classification of capsule opacities. Fibrous plaques were claimed to produce simple attenuation of light entering the eye, whereas Elschnig's pearls and non-uniform clouding produced glare symptoms. ${ }^{12}$ The early studies relied on measurement of glare disability. A simple method of assessing visual deterioration in the face of a glare source is to determine visual acuity with and without a pentorch shone obliquely at the patient's eye. ${ }^{13}$ Other more accurate devices have been developed, but rely on relatively intense light sources to induce glare. ${ }^{7}$ The only methods for forward scatter testing under photopic conditions rely on van den Berg's direct compensation technique. ${ }^{8}$ The development of Lohmann et al.'s computerised system for assessment of forward light scatter has allowed these tests to be rapidly and repeatably carried out.

Studies of variable contrast acuity in patients with transient corneal haze after excimer laser surgery demonstrated that only low contrast acuity was significantly reduced post-operatively. This reduction in visual performance showed no correlation with the measurements of reflected light, but almost absolute correlation with measurements of scattered light. ${ }^{6.7}$ Although measurement of $100 \%$ contrast Snellen visual acuity is the standard clinical assessment of central visual performance, it is of limited value in predicting loss of visual performance in patients with capsular opacification. ${ }^{14}$ It is for this reason that all of our pre-laser patients demonstrated poor results in forward light scatter assessment despite having relatively good Snellen acuities. The results from this study showed that the size of capsulotomy did not appear to influence average Snellen acuity which improved from just less than $6 / 12$ to better than $6 / 9$ in both groups. In terms of the elderly patients that were being treated, this improvement in acuity was felt to be clinically significant.

The initial exposures with the laser were the most likely to produce lens pitting as the capsule could be closely adherent to the posterior surface of the implant. Once the capsulotomy had been started, the tension within the capsule was relieved and a gap often appeared between lens and capsule. This reduced the risk of lens damage. By initiating the capsulotomy at the inferior periphery of the optic, the patients of group B had a reduced chance of laser damage to the central area. In group A the only area being treated was the central zone, i.e. that within the undilated pupil. Pits in the PMMA optic which were visible on slip lamp examination are considerably larger than $1 \mu \mathrm{m}$ and therefore produce mie scatter.

Forward light scatter measurements correlate with loss of low contrast visual acuity and are a sensitive test of central visual performance. ${ }^{7}$ In the pre-operative capsulotomy patients, forward light scatter measurements demonstrated the degradation of visual performance which was not shown by Snellen acuity testing. In the group who 

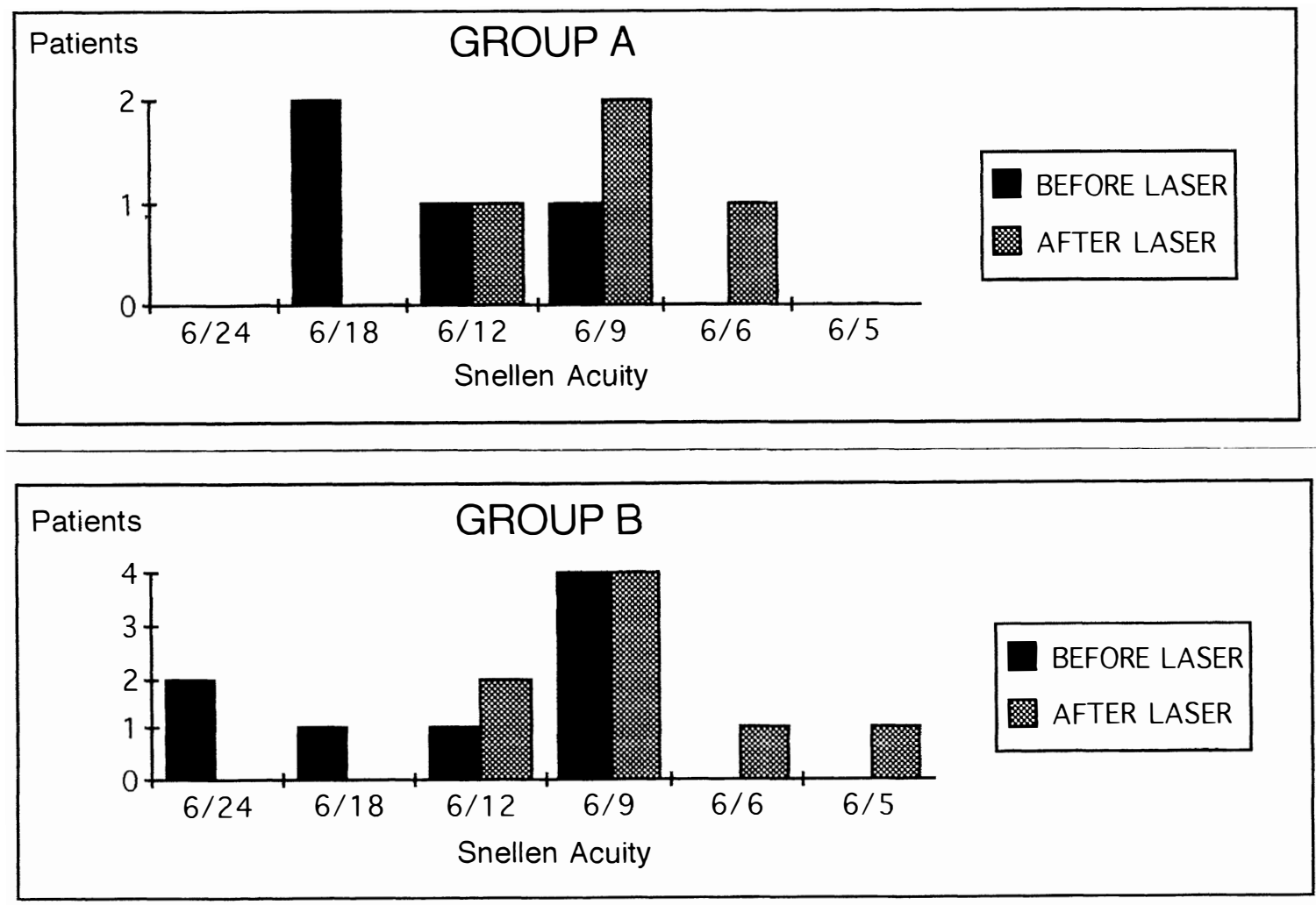

Fig. 1. Snellen acuity in groups $A$ and $B$ before and after $N d: Y A G$ capsulotomy.

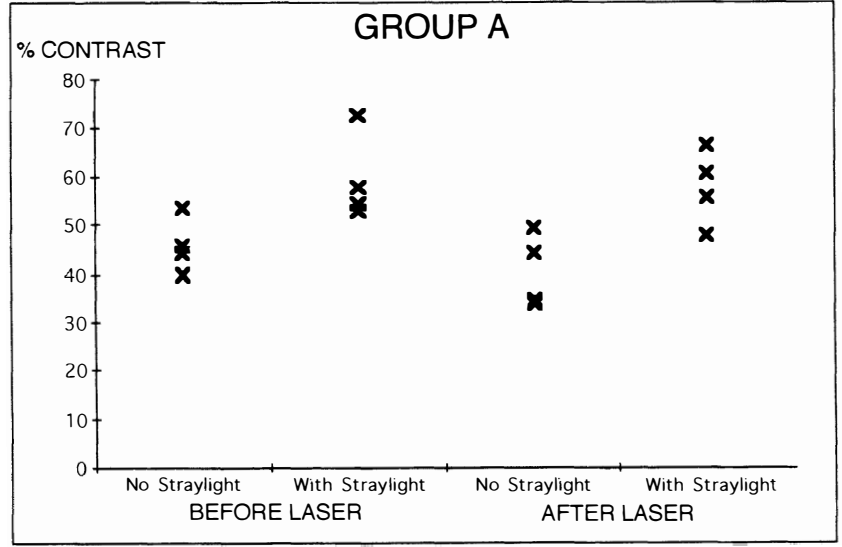

Fig. 2. Forward light scatter measurements in patients of group A before and after Nd:YAG capsulotomy. Each data point represents a single patient's eye.

received wide capsulotomies, there were dramatic improvements in percentage contrast error scores. In the group with small central capsulotomies there was no significant improvement in forward scatter measurements and even the first part of the test (without straylight) failed to improve significantly. This was due to the relatively large amounts of residual capsule which remained within the pupil to produce an irregular artificial entrance aperture and give rise to light scatter. The higher incidence of central lens pitting in the latter group may also have contributed in some cases.

There would appear to be a definite visual benefit in creating a wide capsulotomy. Holladay et al. ${ }^{15}$ theorised



Fig. 3. Forward light scatter measurements in patients of group $B$ before and after Nd:YAG capsulotomy. Each data point represents a single patient's eye.

that a capsulotomy should be at least as large as the scotopic pupil in order to allow it to regulate image intensity and reduce diffraction. The factors that influence the size of a capsulotomy include the tension within the capsule, the pulse energy in relation to the thickness of the capsule and the placement of the laser bursts by the surgeon. If capsule remnants remain within the pupil margin they will continue to produce light scatter and increase background luminance with loss of the contrast of the focussed retinal image. Pitting of the central optic zone by the laser will also produce forward light scatter and the risk may be reduced by initiating the capsulotomy at the lens periphery. 
Creating a larger capsulotomy may theoretically increase the risks of development of cystoid macular oedema (CMO) and retinal detachment after $\mathrm{Nd}$ :YAG capsulotomy. The barrier effect of the posterior capsule prevents vitreous access to the anterior chamber and a small capsulotomy may be a more effective barrier. ${ }^{16-18}$ Clinical studies have not quoted the incidence of vitreous in the anterior chamber or the size of capsulotomies in those patients unfortunate enough to develop such complications after $\mathrm{Nd}$ :YAG laser capsulotomy. The greater number of laser pulses and/or higher total laser energy required to produce a wide capsulotomy may also theoretically carry an increased incidence of complications. This theory is largely unsubstantiated by clinical studies, whereas the benefits in terms of reduction of forward light scatter by capsule remnants are well domonstrated by this study. ${ }^{14.16}$

In conclusion, in order to minimise forward scatter from capsule remnants and reduce the consequent glare disability of the patient, it is recommended that pseudophakic patients with posterior capsular opacification have their pupils dilated during Nd:YAG laser capsulotomy so that a wide capsulotomy can be performed. If laser treatment is undertaken simply to improve Snellen acuity, a small capsulotomy appears to be as effective as a wide capsulotomy.

Key words: Capsulotomy, Glare, Light scatter.

\section{REFERENCES}

1. Courtney P. The National Cataract Surgery Survey. I. Method and descriptive features. Eye 1992;6:487-92.

2. Hodgkins PR, Luff AJ, Morrell AJ, Teye Botchway L, Featherstone TJ, Fielder A. Current practice of extraction and anaesthesia. Br J Ophthalmol 1992;76:323-6.

3. Pizzarello LD. The dimensions of the problem of eye disease among the elderly. Ophthalmology 1987;94: 1191-5.

4. Aron-Rosa DS, Aron JJ, Cohn H. Use of a pulsed pico- second Nd:YAG laser in 6664 cases. Am Intraocul Implant Soc J 1984; 10:35-9.

5. Lohmann CP, Gartry DS, Kerr-Muir MG, Timberlake GT, Fitzke F, Marshall J. 'Haze' in photorefractive keratectomy: its origins and consequences. Lasers Ophthalmol 1991;4:15.

6. Lohmann CP, Gartry DS, Kerr-Muir MG, Timberlake GT, Fitzke F, Marshall J. Corneal haze after excimer laser refractive surgery: objective measurements and functional implications. Eur J Ophthalmol 1991;1:173.

7. Lohmann CP, Fitzke F, O'Brart D, Kerr-Muir M, Timberlake G, Marshall J. Corneal light scattering and visual performance in myopic individuals with spectacles, contact lenses or excimer laser photorefractive keratectomy. Am J Ophthalmol 1993;115:444-53.

8. Van den Berg TJTP. Importance of pathological intraocular light scatter for visual disability. Doc Ophthalmol 1986;61:327.

9. Michelson AA. Studies in optics. University of Chicago Press, Chicago, 1927.

10. Pearce JL. Modern simple extracapsular surgery. Trans Ophthalmol Soc UK 1979;99:170-6.

11. Wilhelmus KR, Emery JM. Posterior capsule opacification following phacoemulsification. Ophthalmol Surg 1980:11: 264-7.

12. Nadler JN. Glare and contrast sensitivity in cataracts and pseudophakia. In: Nadler MP, Miller D, Nadler DJ, editors. Glare and contrast sensitivity for clinicians. Berlin: Springer, 1990.

13. Maltzman BA, Horan C, Rengal A. Penlight test for glare disability of cataracts. Ophthalmic Surg 1988;19:356-8.

14. Steinert RF, Puliafito CA, Kumar SR, Dudak SD, Patel S. Cystoid macular edema, retinal detachment, and glaucoma after Nd:YAG laser posterior capsulotomy. Am J Ophthalmol 1991;112:373-80.

15. Holladay J, Bishop J, Lewis J. The optimal size of a capsulotomy. Am Intraocul Implant Soc J 1985; 11:18-20.

16. Bukelman A, Abrahami S, Oliver M, Pollack A. YAG capsulotomy and cystoid macular edema. Eye 1992;6:35-8.

17. Lewis H, Singer TR, Hanscom TA, Straasma BR. A prospective study of cystoid macular edema after neodymium:YAG laser posterior capsulotomy. Ophthalmology 1987;94:478-82.

18. MacEwen CJ, Baines PS. Retinal detachment following YAG laser capsulotomy. Eye 1989;3:759-63. 